\title{
Factorization Properties of Symbolic Unfoldings of Colored Petri Nets
}

\author{
Thomas Chatain ${ }^{1}$ and Éric Fabre ${ }^{2}$ \\ 1 LSV, ENS Cachan, CNRS, France \\ thomas.chatain@lsv. ens-cachan.fr \\ 2 IRISA/INRIA, Rennes, France \\ Eric.Fabre@irisa.fr
}

\begin{abstract}
The unfolding technique is an efficient tool to explore the runs of a Petri net in a true concurrency semantics, i.e. without constructing all the interleavings of concurrent actions. But even small real systems are never modeled directly as ordinary Petri nets: they use many high-level features that were designed as extensions of Petri nets. We focus here on two such features: colors and compositionality. We show that the symbolic unfolding of a product of colored Petri nets can be expressed as the product of the symbolic unfoldings of these nets. This is a necessary result in view of distributed computations based on symbolic unfoldings, as they have been developed already for standard unfoldings, to design modular verification techniques, or modular diagnosis procedures, for example. The factorization property of symbolic unfoldings is valid for several classes of colored or high-level nets. We derive it here for a class of (high-level) open nets, for which the composition is performed by connecting places rather than transitions.
\end{abstract}

\section{Introduction}

Although they offer a satisfactory representation of concurrency, Petri nets are often difficult to use to model even small real systems. Their drawback is that the state of the system is only represented by the position of the tokens in the places. Consequently, in order to distinguish between the different values that a variable of the system can take, the simplest way is often to use one place per value. Even if more subtle codings are possible, the number of necessary places and transitions becomes very large, or even infinite, which makes the system very hard to comprehend. For this reason several extensions of Petri nets have been proposed, like the well-known colored Petri nets [15].

In [17], Khomenko and Koutny, developed a notion of unfoldings for highlevel Petri nets, which is based on a transformation of the high-level model into a low-level model, in order to reuse the unfolding technique that was developed for low-level models. We call this method expanded unfolding. Our method yields a much more compact structure, where the executions are grouped into symbolic processes that reflect the generic aspect of the model. Symbolic unfoldings were already studied in [11] and [9]. Here we focus on their factorization properties: we 
show how the symbolic unfolding of a large system described as a composition of several components, can be computed from the symbolic unfoldings of the components. Some factorization properties are obtained in [10], but only for high-level processes, not for branching processes or symbolic unfoldings.

We choose a framework where nets are composed via shared places, called interface places or open places when seen from one component. This way of composing nets is very popular because it matches nicely the graphical nature of Petri nets. And it has indeed been introduced in different formalisms. For example in net algebras, where nets can be composed by fusion of places $[6,19,5,14]$. Petri nets with interface of $[21,22]$ use a similar construction. The composition operator of [23] is motivated by the popularity of this kind of compositions and remarks that net process are built by assembling tiles via fusion of places. Closer to our work, [18] defines a partial order semantics for Petri net components that communicate via interface places with an environment. A categorical formalization of open nets was proposed in $[2,3]$, where open net processes are defined, but no unfolding. We discuss later the differences between this work and ours.

The paper is organized as follows. Next section introduces the net family that is used in this paper: a variant of colored Petri nets, enriched with interface places that are used to compose them. We call such nets colored puzzle nets. We introduce an adequate category setting for them and study their compositionality properties. Our morphisms differ clearly from those of $[2,3]$, which is crucial to prepare our main result on unfoldings. Section 3 reviews and adapts a standard expansion procedure for colored nets, that separates colors in order to transform a colored net into an equivalent uncolored one. Section 4 contains the main contribution of the paper. It examines the notion of symbolic unfolding for a colored puzzle net, and studies its relation both to composition and to expansion. It is stated there that the symbolic unfolding of a product puzzle net is the product of the unfoldings of its components.

The detailed proofs can be found in [8].

\section{Colored Puzzle (Petri) Nets and their Composition}

Colored Petri nets were defined by Jensen in [15], as one possible formalism to enhance the flexibility of Petri nets and facilitate the modeling of real systems. In these nets, each token carries some information, traditionally called the color of the token. Of course, transitions can test the color of the tokens they consume, and the color of the created tokens may depend on the color of the consumed tokens. These constraints on the values of the tokens are called guards. In this paper we are not interested in giving a precise syntax to the guards, therefore we simply describe them as sets of possible firing modes, each firing mode assigning a color to each input and output place of the transition. Of course our results about factorization of unfoldings remain valid in presence of syntactical restrictions for the guards.

This section introduces colored puzzle nets, or puzzle nets for short, a variant of colored nets where extra interface places are introduced. These interface places 
are used for connectivity purposes. They model the communication between a component and other components or its environment, as it is the case in reactive systems. As long as the environment is not modeled, it may consume or create tokens at any time in interface places. Then it is meaningless to remember the marking in these places or to test the presence of a token in an interface place when a transition needs it to fire. For this reason interface places have a special status: they are neutral in most operations, like the computation of trajectories, the computation of unfoldings, etc. They only become active when they are connected to another component, in which case they change status and start behaving as ordinary places, and thus impose new constraints on the behavior of the component.

Labels are used for the composition, like in [13] or [1].

\subsection{Colored Puzzle Nets}

A (possibly infinite) set $V$ of colors is given once for all, and is used in all the nets of the paper.

Definition 1 (colored puzzle (Petri) net). A colored puzzle net is a tuple $N \stackrel{\text { def }}{=}\left(P, P^{+}, P^{-}, T\right.$, pre, post, $\left.\Lambda, \lambda, \gamma, M^{0}\right)$ where

$-P, P^{+}$and $P^{-}$are disjoint (possibly infinite) sets of internal places, positive places and negative places respectively (think of magnet polarities); We denote $P^{ \pm} \stackrel{\text { def }}{=} P^{+} \cup P^{-}$the interface places; The polarities are needed when nets are composed by product (see Section 2.3).

$-T$ is a (possibly infinite) set of transitions;

- pre, post $: T \longrightarrow 2^{P \cup P^{ \pm}}$map each transition $t \in T$ to a preset often denoted $\bullet t \stackrel{\text { def }}{=}$ pre $(t)$ and $a$ postset often denoted $t \stackrel{\bullet \text { def }}{=}$ post $(t)$ respectively;

$-\gamma$ maps each transition $t \in T$ to a guard $\gamma(t)$, that is a set of pairs $(\alpha, \beta) \in$ $(\bullet t V) \times\left(t^{\bullet} \longrightarrow V\right)$ called firing modes ;

- the initial marking $M^{0}$ is a multiset of pairs $(p, v) \in\left(P \cup P^{ \pm}\right) \times V$. We sometimes write $M^{0}(p)$ for the multiset of colors in place $p$.

$-\Lambda$ is a label set;

$-\lambda: P \cup P^{ \pm} \longrightarrow \Lambda$ assigns a label to each place, such that only internal places can share the same label, i.e. for all $p_{1}, p_{2} \in P \cup P^{ \pm}$, if $p_{1} \neq p_{2}$ and $\lambda\left(p_{1}\right)=\lambda\left(p_{2}\right)$, then $p_{1}$ and $p_{2}$ are internal places;

The tokens in the interface places $P^{ \pm}$are not considered in the semantics of a single component, since they may be created or consumed freely by the environment. In particular, when a token of an interface place is needed to fire a transition, one must always consider that the token may have been created by the environment. Thus, a marking $M$ for the puzzle net $N$ is a multiset $M: P \times V \rightarrow \mathbb{N} .^{3}$ Transition $t$ is firable from $M$ with firing mode $(\alpha, \beta) \in \gamma(t)$

\footnotetext{
${ }^{3}$ Remark that in the definition of the net, the initial marking is defined also on the interface places. The reason for this is that when we compose two nets, they have to agree on the initial marking of their shared places, that become internal places of the product.
} 


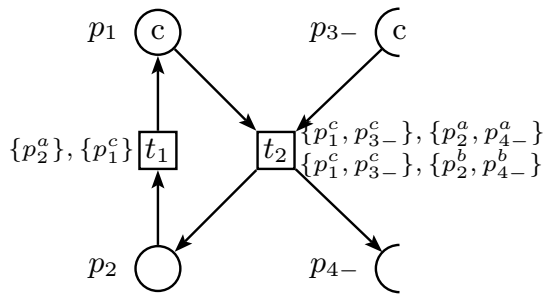

$N_{1}$

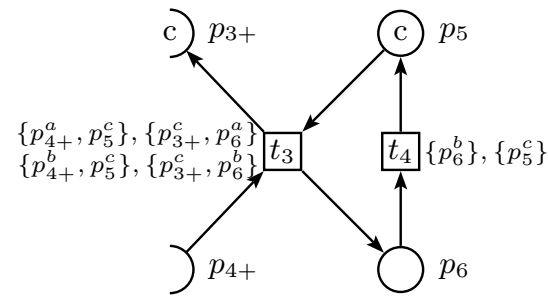

$N_{2}$

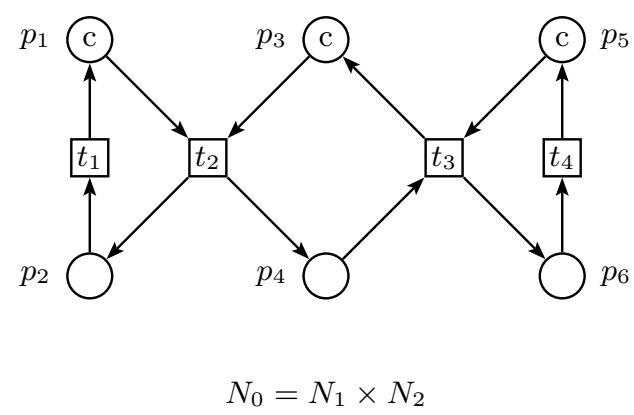

Fig. 1. Two components (on top) and their composition via the product (bottom). The firing modes of the transitions are omitted on the picture of $N_{0}$. They are the same as those of the corresponding transitions of $N_{1}$ and $N_{2}$.

iff $\forall p \in \bullet^{\bullet} t \cap P, M(p, \alpha(p)) \geq 1$. This firing produces the marking

$$
M^{\prime} \stackrel{\text { def }}{=} M-\{(p, \alpha(p)) \mid p \in \bullet t \cap P\}+\left\{(p, \beta(p)) \mid p \in t^{\bullet} \cap P\right\} .
$$

We denote $M[(t, \alpha, \beta)\rangle M^{\prime}$. In other words, $\alpha$ represents the colored tokens that are consumed, and $\beta$ represents those that are produced.

In the standard sequential semantics, a run of $N$ is a sequence of transition firings $M_{0}\left[\left(t_{1}, \alpha_{1}, \beta_{1}\right)\right\rangle M_{1} \ldots M_{n-1}\left[\left(t_{n}, \alpha_{n}, \beta_{n}\right)\right\rangle M_{n}$. Naturally, we will move to a true concurrency semantics for runs, in the sections devoted to unfoldings.

Figure 1 illustrates puzzle nets. Consider $N_{1}$ : it has two internal places $p_{1}$ and $p_{2}$, and two interface places $p_{3}, p_{4}$ that are both of negative sign (mentioned in subscript). The initial marking has placed a token of color $c$ in $p_{1}$ and $p_{3}$, while $p_{2}$ and $p_{4}$ are empty. The transition modes are represented close to each transition. $t_{1}$ has a single firing mode: it consumes a token of color $a$ in $p_{2}$, and produces a token of color $c$ in $p_{1}$. By contrast, $t_{2}$ has two firing modes. Both consume a token of color $c$ in $p_{1}$ and in $p_{3}$. But the first mode places a token of color $a$ in $p_{2}$ and $p_{4}$, while the second mode places a token of color $b$ in these places. 
Consider now $N_{0}$, that also obeys the above constraints. Only two maximal executions are possible. $t_{2}$ fires first and produces two tokens of the same color ( $a$ or $b$ ), one in $p_{2}$ and one in $p_{4}$. Then, if these tokens are of color $a, t_{1}$ and $t_{3}$ can fire concurrently and the net stops; otherwise, $t_{3}$ and $t_{4}$ fire in sequence. Notice that because of the guards, no execution contains both $t_{1}$ and $t_{4}$. Without colors these two transitions could have fired concurrently. This phenomenon will be formalized later as the notion of color conflict.

\subsection{Morphisms}

Before moving to the definition of composition for colored puzzle nets, we need the extra notion of morphism between two colored puzzle nets. Morphisms are relations between nets that ensure the preservation of the behaviors.

Definition 2 (morphism). Let $N_{1}$ and $N_{2}$ be two nets such that $\Lambda_{2} \subseteq \Lambda_{1}$ (we add subscript $i$ to elements of $N_{i}$ ). A morphism $\phi$ from $N_{1}$ to $N_{2}$ is a pair $\left(\phi^{T}, \phi^{P}\right)$ of partial functions (the symbol $*$ is used when the function is undefined)

$$
\begin{aligned}
\phi^{T}: & T_{1} \longrightarrow T_{2} \cup\{*\} \\
\phi^{P}: & \left\{\begin{array}{l}
P_{1} \longrightarrow P_{2} \cup P_{2}^{ \pm} \cup\{*\} \\
P_{1}^{+} \longrightarrow P_{2}^{+} \cup\{*\} \\
P_{1}^{-} \longrightarrow P_{2}^{-} \cup\{*\}
\end{array}\right.
\end{aligned}
$$

such that

$$
\begin{aligned}
& -\forall p_{1} \in P_{1} \cup P_{1}^{ \pm} \quad\left\{\begin{array}{l}
\phi^{P}\left(p_{1}\right)=* \text { iff } \lambda_{1}\left(p_{1}\right) \notin \Lambda_{2} \\
\lambda_{1}\left(p_{1}\right) \in \Lambda_{2} \Longrightarrow \lambda_{2}\left(\phi^{P}\left(p_{1}\right)\right)=\lambda_{1}\left(p_{1}\right)
\end{array}\right. \\
& -\forall t_{1} \in T_{1} \quad \phi^{T}\left(t_{1}\right)=* \Longrightarrow \phi^{P}\left(\bullet t_{1} \cup t_{1} \bullet\right) \subseteq P_{2}^{ \pm} \cup\{*\} ; \\
& - \text { for all } t_{1} \in T_{1} \text { such that } \phi^{T}\left(t_{1}\right)=t_{2} \neq *, \\
& \bullet * \notin \phi^{P}\left(\bullet t_{1} \cup t_{1} \bullet\right) \\
& \bullet \text { the restriction of } \phi^{P} \text { to } t_{1} \text { is a bijection from } \bullet t_{1} \text { to } t_{2} \\
& \bullet \text { the restriction of } \phi^{P} \text { to } t_{1} \bullet \text { is a bijection from } t_{1} \bullet \text { to } t_{2} \bullet \\
& \bullet \forall\left(\alpha_{1}, \beta_{1}\right) \in \gamma_{1}\left(t_{1}\right), \exists\left(\alpha_{2}, \beta_{2}\right) \in \gamma_{2}\left(t_{2}\right): \forall p_{1} \in t_{1}, \alpha_{2}\left(\phi^{P}\left(p_{1}\right)\right)=\alpha_{1}\left(p_{1}\right), \\
& \\
& \text { and } \forall p_{1} \in t_{1} \bullet \beta_{2}\left(\phi^{P}\left(p_{1}\right)\right)=\beta_{1}\left(p_{1}\right), \\
& -\forall p_{2} \in P_{2} \cup P_{2}^{ \pm}, \quad M_{2}^{0}\left(p_{2}\right)=\sum_{p_{1}: \phi^{P}\left(p_{1}\right)=p_{2}} M_{1}^{0}\left(p_{1}\right) .
\end{aligned}
$$

In words, $\phi^{P}$ is defined exactly on places that carry a label of the image net, and preserves these place labels. $\phi^{P}$ also preserves the polarity of an interface place, and may assign a polarity to an internal place. This change of status must occur for places connected to a transition $t_{1}$ that is removed by $\phi^{T}$, unless if such places vanish through $\phi^{P}$. When a transition $t_{1}$ is preserved by $\phi^{T}$, all its connected places are preserved as well, and firing modes of this transition $t_{1}$ are mapped into the modes of its image $t_{2}$. Notice in particular that $N_{1}$ and $N_{2}$ may be identical up to their firing modes, and the identity mapping is then a morphism as soon as $\gamma_{1} \subseteq \gamma_{2}$.

From this definition, it is clear that a run of $N_{1}$ is naturally mapped by $\phi$ into a run of $N_{2}$. 
The composition of two morphisms $\phi_{1} \stackrel{\text { def }}{=}\left(\phi_{1}^{T}, \phi_{1}^{P}\right)$ from $N_{1}$ to $N_{2}$ and $\phi_{2} \stackrel{\text { def }}{=}$ $\left(\phi_{2}^{T}, \phi_{2}^{P}\right)$ from $N_{2}$ to $N_{3}$ is $\phi_{2} \circ \phi_{1} \stackrel{\text { def }}{=}\left(\phi_{2}^{T} \circ \phi_{1}^{T}, \phi_{2}^{P} \circ \phi_{1}^{P}\right)$. The identity morphism for $N$ is $1_{N} \stackrel{\text { def }}{=}\left(1_{T}, 1_{P \cup P^{ \pm}}\right)$.

Theorem 1. The family of colored puzzle nets equipped with the above notion of morphism forms a category.

Proof sketch. Associativity and identity are straightforward. The proof of the composition can be found in [8].

To simplify the notations we often write $\phi$ instead of $\phi^{T}$ or $\phi^{P}$. We also denote by $N_{1} \sim N_{2}$ the fact that $N_{1}$ and $N_{2}$ are isomorphic, i.e. the existence of two morphisms $\phi_{12}$ from $N_{1}$ to $N_{2}$ and $\phi_{21}$ from $N_{2}$ to $N_{1}$ such that $\phi_{21} \circ \phi_{12}=1_{N_{1}}$ and $\phi_{12} \circ \phi_{21}=1_{N_{2}}$.

Comparison with the category of open nets $[2,3]$. Forgetting high-level features like colors and guards, our puzzle nets are close to the open nets proposed in $[2,3]$. But the morphisms between them are quite different. Apart from technical aspects (we use partial functions, rather than total ones), a significant difference is that they preserve runs in the opposite direction: in our category, a morphism from $N_{1}$ to $N_{2}$ maps every run of $N_{1}$ to a run of $N_{2}$ (i.e. $N_{2}$ simulates $N_{1}$ ), whereas in open nets, a morphism from $N_{1}$ to $N_{2}$ maps every run of $N_{2}$ to a run of $N_{1}$ (i.e. $N_{1}$ simulates $N_{2}$ ).

The composition operations defined for open net and for puzzle nets are very similar in their principle: they both amount to identifying places that carry the same label. In the case of open nets, this labeling comes from the injection of a common interface net into the two components that must be assembled. In both categories, the two components that are assembled both simulate the resulting composed net. However, as morphisms and simulation relations do not have the same directions in the two settings, the composition is expressed as a pushout for open nets (a colimit), and as a product for puzzle nets (a limit).

This difference becomes crucial when coming to the construction of processes, and more generally unfoldings. In the category of open nets, there is a morphism from a process to the net $N$, expressing that this process simulates net $N$. By contrast, in the category of puzzle nets, the similar morphism from the (branching) process $O$ to the net $N$ expresses that net $N$ simulates the branching process $O$, or more generally the unfolding of $N$. This choice of direction is crucial to obtain a universal property on unfoldings, which is the key to transport compostion operations from nets to unfoldings. Notice that this morphism architecture reproduces the one followed in [24].

\subsection{Product}

The meaning of interface places appears in the compositon of puzzle nets, that we are going to express as a categorical product. The composition mechanism is governed by the following idea, that mimics the one proposed for open nets $[2,3]$ : 
when two components are connected, interface places with identical labels will be merged, provided they have complementary polarities, just like magnets. Once two places are merged, the resulting pair becomes an ordinary internal place of the composed (product) net, i.e. the polarity vanishes. Notice that this notion of polarity has no relation with any idea of input or output place: interface places can communicate with the environment in any direction.

Polarities represent the part of the interface place that is owned by each component. This is why morphisms must respect polarities: an interface place can only be simulated by an interface place with the same polarity. It would be possible to deal with more than two polarities, meaning that some interface place could be shared by more than two components. An interface place could then be seen as a pie-chart, each component owning a part of the pie-chart; as long as the pie-chart would not be full, the place would keep its status of interface. When full, the place would become an internal place. This idea of multiple polarities can help one convince himself that interface places do not need to be used only as inputs or only as outputs.

Definition 3 (product). Let $N_{1}$ and $N_{2}$ be two nets such that

$$
\begin{aligned}
& -\forall p_{1}, p_{2} \quad \lambda_{1}\left(p_{1}\right)=\lambda_{2}\left(p_{2}\right) \Longrightarrow\left\{\begin{array}{l}
\left(p_{1}, p_{2}\right) \in\left(P_{1}^{+} \times P_{2}^{-}\right) \cup\left(P_{1}^{-} \times P_{2}^{+}\right) \\
M_{1}^{0}\left(p_{1}\right)=M_{2}^{0}\left(p_{2}\right)
\end{array}\right. \\
& -\forall t_{i} \in T_{i} \quad\left(\bullet \bullet_{i} \cup t_{i}^{\bullet}\right) \cap P_{i} \neq \emptyset .
\end{aligned}
$$

We define their product $N_{0}$, denoted $N_{1} \times N_{2}$ and the associated morphisms $\pi_{1}$ and $\pi_{2}$ as:

$$
\begin{aligned}
& \text { places: } \\
& P_{0} \stackrel{\text { def }}{=}\left(P_{1} \times\{*\}\right) \cup\left(\{*\} \times P_{2}\right) \cup\left\{\left(p_{1}, p_{2}\right) \mid \lambda_{1}\left(p_{1}\right)=\lambda_{2}\left(p_{2}\right)\right\} \\
& P_{0}^{+} \stackrel{\text { def }}{=}\left\{\left(p_{1}, *\right) \mid p_{1} \in P_{1}^{+} \wedge \lambda_{1}\left(p_{1}\right) \notin \Lambda_{2}\right\} \cup\left\{\left(*, p_{2}\right) \mid p_{2} \in P_{2}^{+} \wedge \lambda_{2}\left(p_{2}\right) \notin \Lambda_{1}\right\} \\
& P_{0}^{-} \stackrel{\text { def }}{=}\left\{\left(p_{1}, *\right) \mid p_{1} \in P_{1}^{-} \wedge \lambda_{1}\left(p_{1}\right) \notin \Lambda_{2}\right\} \cup\left\{\left(*, p_{2}\right) \mid p_{2} \in P_{2}^{-} \wedge \lambda_{2}\left(p_{2}\right) \notin \Lambda_{1}\right\} \\
& \pi_{i}^{P}\left(\left(p_{1}, p_{2}\right)\right) \stackrel{\text { def }}{=} p_{i} \text { (even when } p_{i}=* \text { ). } \\
& \text { labels: } \\
& \Lambda_{0} \stackrel{\text { def }}{=} \Lambda_{1} \cup \Lambda_{2} \\
& \lambda_{0}\left(\left(p_{1}, p_{2}\right)\right) \stackrel{\text { def }}{=} \lambda_{i}\left(p_{i}\right) \text { when } p_{i} \neq * \text {. } \\
& \text { Notice that the restriction of } \pi_{i}^{P} \text { to } \lambda_{0}^{-1}\left(\Lambda_{i}\right) \text { is a bijection to } P_{i} \cup P_{i}^{ \pm} \text {. } \\
& \text { initial marking: } \\
& M_{0}^{0}\left(\left(p_{1}, p_{2}\right)\right) \stackrel{\text { def }}{=} M_{i}^{0}\left(p_{i}\right) \text { when } p_{i} \neq * \\
& \text { transitions: } \\
& T_{0} \stackrel{\text { def }}{=}\left(T_{1} \times\{*\}\right) \cup\left(\{*\} \times T_{2}\right) \\
& \pi_{i}^{T}\left(\left(t_{1}, t_{2}\right)\right) \stackrel{\text { def }}{=} t_{i} \text { (even when } t_{i}=* \text { ) } \\
& \text { - }\left(t_{1}, t_{2}\right) \stackrel{\text { def }}{=} \pi_{i}{ }^{-1}\left(\bullet t_{i}\right) \text { and }\left(t_{1}, t_{2}\right)^{\bullet} \stackrel{\text { def }}{=} \pi_{i}{ }^{-1}\left(t_{i} \bullet\right) \text { when } t_{i} \neq * \\
& \gamma\left(t_{0}\right) \stackrel{\text { def }}{=}\left\{\left(\alpha \circ \pi_{i}^{-1} \bullet_{0}, \beta \circ \pi_{i \mid t_{0}}^{-1}\right) \mid(\alpha, \beta) \in \gamma\left(\pi_{i}\left(t_{0}\right)\right)\right\} \text { when } \pi_{i}\left(t_{0}\right) \neq *
\end{aligned}
$$

Observe that this composition takes the disjoint union of transitions, by contrast with several alternate notions of product for Petri nets that rather synchronize transitions. Since transitions remain private, their flow is preserved, as well as 


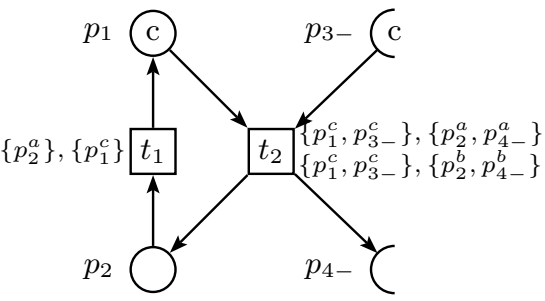

$N_{1}$

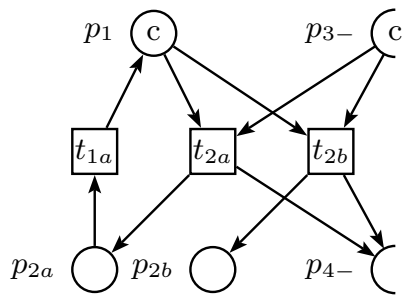

$\operatorname{Exp}\left(N_{1}\right)$

Fig. 2. A colored puzzle net (left) and its expansion (right).

their firing modes, up to the reshaping of place names performed by the composition.

Figure 1 illustrates the composition by product. Places labeled $p_{3}$ in $N_{1}$ and $N_{2}$ are merged in the product, because they have complementary polarities (the product would be undefined if they had identical polarities). The same holds for places labeled $p_{4}$. So nets $N_{1}$ and $N_{2}$ are assembled by these interface places, that now change their status to internal places.

Theorem 2. Definition 3 corresponds to the categorical product in the category of puzzle nets (Definitions 1 and 2).

Proof sketch. One has to check that $N_{0}$ is a net: the non-trivial part is to show that only internal places share labels. Then we show easily that $\pi_{i}$ is a morphism from $N_{0}$ to $N_{i}$.

Finally it remains to check the universal property of the product in this category: for any $N$ and any pair of morphisms $\phi_{i}: N \longrightarrow N_{i}$, there exists a unique morphism $\psi$ from $N$ to $N_{0}=N_{1} \times N_{2}$ that makes the diagram commutative, i.e. that satisfies $\phi_{i}=\pi_{i} \circ \psi$. This $\psi$ is necessarily defined by:

$$
\forall x \in P \cup P^{ \pm} \cup T \quad \psi(x) \stackrel{\text { def }}{=} \begin{cases}* & \text { if } \phi_{1}(x)=\phi_{2}(x)=* \\ \left(\phi_{1}(x), \phi_{2}(x)\right) & \text { otherwise. }\end{cases}
$$

It is straightforward to check that $\psi$ satisfies Definition 2.

\section{Expansion}

There exists a classical method to expand colored Petri nets into ordinary (or low-level) nets, which sometimes motivated the use of high-level nets as convenient generators of low-level models. This expansion operation is called unfolding by some authors [6]. In this paper, we prefer to call it expansion, and reserve the term unfolding for its standard meaning, since both operations will be simultaneously applied to colored puzzle nets. 
Definition 4 (expanded net). An expanded net is a colored puzzle net $N$ such that:

$-\forall t \in T, \quad|\gamma(t)|=1$ (the unique element of $\gamma(t)$ is denoted $\left(\alpha_{t}, \beta_{t}\right)$ )

$-\forall p \in P \quad\left|\left\{\alpha_{t}(p) \mid p \in \bullet^{\bullet} t\right\} \cup\left\{\beta_{t}(p) \mid p \in t^{\bullet}\right\}\right|=1$ (the unique color in this set is denoted $\operatorname{col}(p))$

In other words, transitions have a single firing mode, and places can carry tokens of a single color of $V$, which coincides with the mode of all connected transitions. Notice that the second condition doesn't apply to interface places: they are not expanded into their different colors, which corresponds to the idea that a puzzle net should not restrict the set of colors in a place that will eventually be shared with another component. Anticipating a little, interface places will not be duplicated either by the unfolding procedure.

Definition 5 (expansion). Given a colored puzzle net $N$, we define its expansion $\operatorname{Exp}(N) \stackrel{\text { def }}{=}\left(P^{\prime}, P^{+}, P^{-}, T^{\prime}\right.$, pre $e^{\prime}$, post $\left.^{\prime}, \Lambda, \lambda^{\prime}, \gamma^{\prime}, M^{0^{\prime}}\right)$ and the associated compression morphism $\chi_{N}: \operatorname{Exp}(N) \rightarrow N$ as:

places:

$$
\begin{aligned}
& P^{+} \text {and } P^{-} \text {are the sets of interface places of } N \\
& P^{\prime} \stackrel{\text { def }}{=}\{(p, v) \in P \times V \mid \exists t \in T,(\alpha, \beta) \in \gamma(t) \\
& \operatorname{col}(p, v)=v \\
& \left.\qquad\left(p \in{ }^{\bullet} t \wedge \alpha(p)=v\right) \vee\left(p \in t^{\bullet} \wedge \beta(p)=v\right)\right\}
\end{aligned}
$$

transitions and flow:

$T^{\prime} \stackrel{\text { def }}{=}\{(t,(\alpha, \beta)) \mid t \in T \wedge(\alpha, \beta) \in \gamma(t)\}$

$\bullet(t,(\alpha, \beta)) \stackrel{\text { def }}{=}\left\{(p, \alpha(p)) \mid p \in \bullet^{\bullet} t \cap P\right\} \cup\left(\bullet^{\bullet} t \cap P^{ \pm}\right)$

$(t,(\alpha, \beta)) \stackrel{\text { def }}{=}\left\{(p, \beta(p)) \mid p \in t^{\bullet} \cap P\right\} \cup\left(t^{\bullet} \cap P^{ \pm}\right)$

initial marking:

$\forall(p, v) \in P^{ \pm} \times V, \quad M^{0^{\prime}}(p, v)=M^{0}(p, v)$

$\forall p^{\prime}=(p, v) \in P^{\prime}, \quad \forall v^{\prime} \in V, \quad M^{0^{\prime}}\left(p^{\prime}, v^{\prime}\right)= \begin{cases}M^{0}(p, v) & \text { if } v=v^{\prime} \\ 0 & \text { otherwise }\end{cases}$

firing modes: for all $t^{\prime}=(t,(\alpha, \beta)) \in T^{\prime}$

$\forall p^{\prime}=(p, v) \in P^{\prime}, \quad \begin{cases}\alpha_{t^{\prime}}^{\prime}\left(p^{\prime}\right) \stackrel{\text { def }}{=} v & \text { iff } p^{\prime} \in \bullet t^{\prime} \\ \beta_{t^{\prime}}^{\prime}\left(p^{\prime}\right) \stackrel{\text { def }}{=} v & \text { iff } p^{\prime} \in t^{\prime}\end{cases}$

$\forall p \in P^{ \pm}, \quad \alpha_{t^{\prime}}^{\prime}(p) \stackrel{\text { def }}{=} \alpha(p)$ and $\beta_{t^{\prime}}^{\prime}(p) \stackrel{\text { def }}{=} \beta(p)$

labels and morphism:

$\Lambda$ is the label set of $N$

$\forall p \in P^{ \pm}, \quad \chi_{N}(p) \stackrel{\text { def }}{=} p$ and $\lambda^{\prime}(p) \stackrel{\text { def }}{=} \lambda(p)$

$\forall p^{\prime}=(p, v) \in P^{\prime}, \chi_{N}\left(p^{\prime}\right) \stackrel{\text { def }}{=} p$ and $\lambda^{\prime}\left(p^{\prime}\right) \stackrel{\text { def }}{=} \lambda(p)$

$\forall t^{\prime}=(t,(\alpha, \beta)) \in T^{\prime}, \quad \chi_{N}\left(t^{\prime}\right) \stackrel{\text { def }}{=} t$

Proposition 1. The expansion of a colored puzzle net yields an expanded net, and the mapping $\chi_{N}: \operatorname{Exp}(N) \rightarrow N$ is a (compression) morphism of colored puzzle nets. 
Proof. We have to show that $\operatorname{Exp}(N)$ is an expanded net. The only non-trivial part here concerns the condition about the colors in internal places (second item of Definition 4): it is ensured by the definition of the internal places $P^{\prime}$ through the pre- and post-sets of the expanded transitions.

Checking that $\chi_{N}$ is a morphism from $\operatorname{Exp}(N)$ to $N$ is straightforward.

Theorem 3 (expansion). The Exp functor establishes a coreflection between the category of colored puzzle nets, and the full subcategory of expanded nets.

Proof sketch. We have to prove the universal property of each expanded net $\operatorname{Exp}(N)$, associated to its compression morphism $\chi_{N}$, i.e. for every morphism $\phi$ from an expanded net $N^{\prime}$ to a puzzle net $N$, there exists a unique morphism $\psi$ from $N^{\prime}$ to $\operatorname{Exp}(N)$ such that $\phi=\chi_{N} \circ \psi$. If such a morphsim $\psi$ exists, this latter condition imposes the following definition:

$-\psi(x)=* \quad$ iff $\quad \phi(x)=*$

- $\phi(p) \in P^{ \pm} \Longrightarrow \psi(p) \stackrel{\text { def }}{=} \phi(p)$

$-\phi(p) \in P \Longrightarrow \psi(p) \stackrel{\text { def }}{=}(\phi(p), \operatorname{col}(p))$

$-\psi(t) \stackrel{\text { def }}{=}\left(\phi(t),\left(\alpha_{t} \circ \phi_{\mid \bullet t}^{-1}, \beta_{t} \circ \phi_{\mid t^{\bullet}}^{-1}\right)\right)$

It remains to show that $\psi$ does satisfy the conditions for being a morphism from $N^{\prime}$ to $\operatorname{Exp}(N)$, and that $\phi=\chi \circ \psi$.

A nice consequence of this coreflection is that expansion and product commute. One has first that there exists a product in the subcategory of expanded nets, defined by $N_{1} \times_{E} N_{2}=\operatorname{Exp}\left(N_{1} \times N_{2}\right)$, where $N_{1} \times N_{2}$ is the product in the sense of colored puzzle nets. Notice that the expansion operator in the right hand side term is necessary in order to expand as well the interface places that become internal after the standard product of colored nets. Now, since products are special cases of categorical limits (theorem 2), and given that limits are preserved by functors that have a left adjoint, one has:

$$
\operatorname{Exp}\left(N_{1} \times N_{2}\right) \sim \operatorname{Exp}\left(N_{1}\right) \times_{E} \operatorname{Exp}\left(N_{2}\right)
$$

This relation would be tedious to prove directly, so it is interesting to obtain it by standard structural derivations (that exactly reproduce those of [24] in their structure).

\section{$4 \quad$ Symbolic Unfolding and its Properties}

Unfoldings provide a compact data structure to encode sets of runs of a Petri net in a true concurrency semantics. By their ability to avoid the combinatorial explosion due to the interleaving of concurrent events, they are particularly suited to analyse properties of distributed systems. And they have indeed been used in this sense, to check the absence of deadlocks, or for reachability analysis. Unfoldings have been defined for ordinary (low-level) safe nets, and more generally for semi-weighted nets. Some authors have extended this construction 


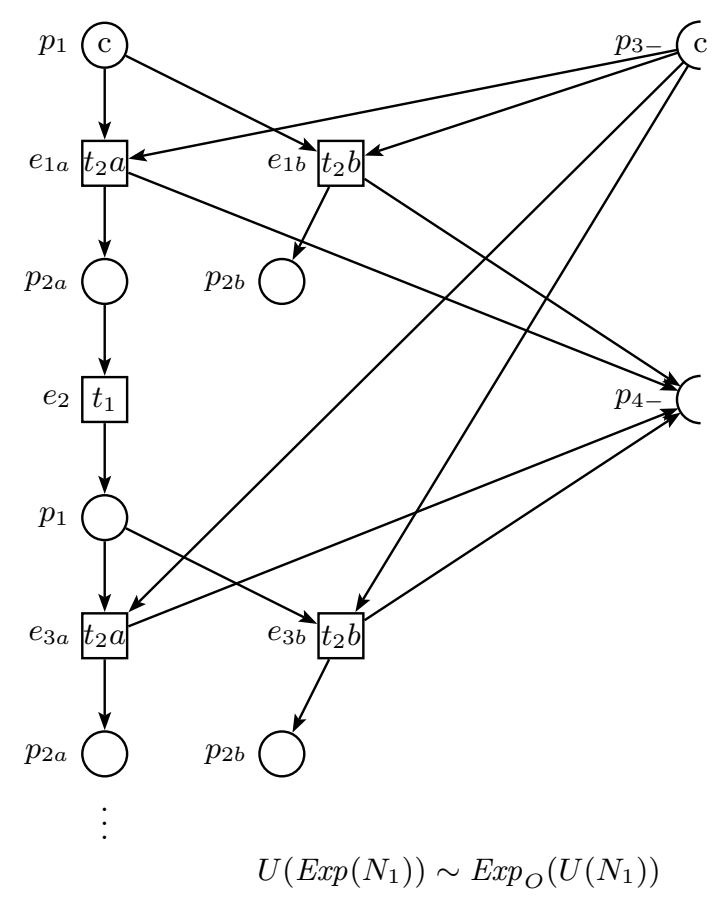

Fig. 3. The expanded unfolding of the colored puzzle net $N_{1}$ of Figure 1 .

to colored (or high-level) nets by first performing what is called an expansion in this paper, and then applying a standard unfolding procedure, as illustrated in Fig. 3. This is the approach of $[17,16]$ for a model of high-level Petri nets called M-nets [7].

In this paper, we propose to go further in this direction, and define directly the unfolding of a colored puzzle net as a symbolic unfolding, that is as some form of "colored puzzle branching process." The principles are the same as in [11] or [9], where we also advocated the interest of symbolic unfoldings for the diagnosis of distributed systems. We then study the relations between symbolic unfolding and expansion. The main contribution of this section is the derivation of a factorization property of symbolic unfoldings, as it was already derived by Winskel [24] for ordinary unfoldings. Namely, the symbolic unfolding of a product of puzzle nets is the product (in a specific sense) of the symbolic unfoldings of the components. This derivation follows the same principles as for the expansion, by producing an adequate coreflection between categories.

Generic Executions for a Generic Model. In high-level processes we definitely want to benefit from the generic aspects of the colored puzzle net that we are considering. Indeed in a colored puzzle net, if several states share the same marking (that is the tokens are in the same places but do not carry the same values), 
we can view these states as instances of a generic family of states. Similarly each transition is a generic representation of a family of actions, that differ only by the values/colors of the tokens that are consumed and created. And we consider that grouping several states into a generic state or several actions into a high-level transition, results from a choice that was done when the system was modeled.

With respect to this choice, we can identify families of executions of a colored puzzle net $N$ based on the generic aspects related to its places and transitions. To do this, our approach is based on the fact that each execution of $N$ can be mapped to an execution of the underlying low-level Petri net obtained by simply removing the colors and the guards.

Definition 6 (symbolic occurrence net). $A$ symbolic occurrence net is a colored puzzle net $O \stackrel{\text { def }}{=}\left(B, P^{+}, P^{-}, E\right.$, pre, post $\left., \Lambda, \lambda, \gamma, M^{0}\right)$ where the internal places, denoted $B$ here, are called conditions and the transitions, denoted $E$ here, are called events, which satisfies:

$-\rightarrow^{+}$is acyclic, where $\rightarrow$ denotes the causality relation, defined as $\left(e_{1} \rightarrow\right.$ $\left.e_{2}\right) \stackrel{\text { def }}{\Longleftrightarrow}\left(e_{1} \bullet \cap e_{2} \cap B \neq \emptyset\right)$. Notice that the interface places induce no causality, since they are not "unfolded".

$$
\begin{aligned}
& -\forall b \in B \\
& \left\{\begin{array}{l}
\left.\sum_{v \in V} M^{0}(b, v)=0 \wedge \exists ! e \in E \quad b \in e^{\bullet} \quad \text { (then this } e \text { is denoted } \bullet b\right) \\
\left.\vee \sum_{v \in V} M^{0}(b, v)=1 \wedge \nexists e \in E b \in e^{\bullet} \quad \text { (then we define } \bullet \stackrel{\text { def }}{=} \perp\right)
\end{array}\right. \\
& -\forall e \in E \quad\left\{\begin{array}{l}
\lceil e\rceil \stackrel{\text { def }}{=}\left\{f \in E \mid f \rightarrow^{*} e\right\} \text { is finite } \\
\nexists e_{1}, e_{2} \in\lceil e\rceil e_{1} \neq e_{2} \wedge \\
\text { valid_colorings }(\lceil e\rceil) \neq \emptyset
\end{array} e_{1} \cap e_{2} \cap B \neq \emptyset\right.
\end{aligned}
$$

where, for every set $F$ of events, valid_colorings $(F)$ denotes the set of colorings Col $:\left(\bullet F \cup F^{\bullet}\right) \cap B \longrightarrow V$ of the input and output conditions of the events in $F$, that are compatible with the firing modes of these events and with the color of the tokens in the initial conditions:

$$
\begin{cases}\forall e \in F \quad \exists(\alpha, \beta) \in \gamma(e) \quad\left(\alpha \mid \cdot e \cap B, \beta_{\mid e \cdot \cap B}\right)=\left(\operatorname{Col}_{\mid \bullet \bullet \cap B}, \operatorname{Col}_{\mid e \bullet \cap B}\right) \\ \forall b, v \quad M^{0}(b, v)=1 \Longrightarrow \operatorname{Col}(b)=v .\end{cases}
$$

In an occurrence net, places are usually called conditions, and transitions are called events. Concerning conditions, the second point in the definition requires that each of them is created (i.e. immediately preceded) by a unique event, or it is minimal, and marked with a single token. On events, the requirements are standard. The first line expresses the well-foundedness (configurations are finite), and the second line expresses that no node should be in (structural) self-conflict. Or equivalently that there is no immediate conflict in the past of each event.

Treatment of Interface Places. Note that the interface places are not treated like the internal places. The idea is that only the behaviour of the component is represented in the occurrence net, and no assumption is made about the components it will be connected to. In particular, the events can freely use tokens from the interface places, considering that they may be filled with any number of tokens of any kind. 
Color Conflict. Processes of a colored Petri net have to satisfy both structural conditions and conditions imposed by the guards on the possible values for the firing modes. The structural conditions only depend on the underlying low-level process and express:

- the causal dependencies (noted $\rightarrow^{+}$), which induce a partial ordering on events,

- the structural conflicts, identified by the consumption of a condition by two different events, which implies that these two events cannot occur in the same execution, as well as their successors for the causal relation (conflict is inherited by causality),

- the concurrency relation: when two events are neither causally related nor in conflict, they are said to be concurrent. They can then occur in any order in an execution.

But these structural conditions are not sufficient when we deal with symbolic occurrence nets: a set of events can be made incompatible by the fact that there exists no suitable value for the firing modes of the events in their past, even if they would be concurrent in the underlying low-level process.

We can say that a set $E$ of events of $O$ are in color conflict if they are not in conflict, but the constraints on the values of the firing modes, coming from the guards of the transitions, prevent the events of $E$ to appear in the same process of $N$.

Observe that in the example of Figure 5 the symbolic unfolding of $N_{0}$ is finite because $e_{2}$ and $e_{5}$ are in color conflict: they impose contradictory constraints on the token in $p_{4}$ after $e_{1}$ fires: $e_{2}$ can fire only if it is $a$, but $e_{5}$ only if it is $b$. Nevertheless, without colors, the unfolding would have been infinite.

Unlike the structural conflict due to the consumption of a single condition by several events, color conflicts are not binary in general. That is, the minimal sets of events that are in conflict may have more than two elements.

In the definition of symbolic occurrence nets, color conflicts are treated in the valid_colorings function, which deals both with colors and with the symbolic aspects. The existence of a valid coloring expresses that, for each event $e$, there is a way of coloring the configuration $\lceil e\rceil$ leading to $e$ in a coherent manner. This coloring assigns a color to every condition and ensures that these colors are compatible with the firing modes of the events.

We are now equipped to define symbolic unfoldings $U(N)$ of a colored puzzle net $N$. A minor and classical restriction on the structure of a Petri net is necessary in order to define its unfolding: we require that every transition consumes at least one token from an internal place. Interface places do not really participate in the unfolding and are not duplicated. Moreover the initial marking must not contain more than one token per place (even if they have different colors).

Remark: when dealing with weighted arcs, another condition is also required: the output arcs of the transitions must be simple, i.e. no transition should produce more than one token per output place. These nets are called "semi-weighted 
nets" in $[20,4]$. Here we did not consider weighted arcs, so this condition is satisfied by construction.

In our definition of symbolic unfoldings, we use the canonical coding of events and conditions introduced in [12], based on a backward chaining principle. As the unfoldings are occurrence nets, each condition $b \in B$ is created by a single event $e$ denoted $\bullet b$, if we take the convention that $\bullet b$ may be either an event of $E$ or the virtual initial event $\perp$ when $b$ represents a token of the initial marking. Moreover, as each event $e$ of the unfolding of a net $N$ represents an occurrence of a transition $t$ of $N$, then the output conditions of $e$ represent the tokens created in the internal places of $t^{\bullet} \cap P$. Thus each of these conditions is identified by a pair $(e, p)$ with $p \in t^{\bullet} \cap P$. Similarly, every event $e$ of the unfolding is itself a pair $(C, t)$ where $t$ is a transition of $N$, and $C \subseteq B$ is the set of conditions that are consumed by $e$. As an example, in Figure 5 , the coding of the events is written on the right.

The folding morphism $\phi_{N}$ from the unfolding $U(N)$ to the net $N$ reflects also the correspondence between the events (respectively conditions) of the unfolding and the transitions (respectively places) of the net. It is defined as:

$-\forall e=(C, t) \in E \quad \phi_{N}(e)=t$,

$-\forall b=(e, p) \in B \quad \phi_{N}(b) \stackrel{\text { def }}{=} p$ and

$-\forall p \in P^{ \pm} \quad \phi_{N}(p)=p$, since the interface places are not unfolded.

Definition 7 (symbolic unfolding). Let $N$ be a colored puzzle net such that

- $\forall p \in P \quad \sum_{v \in V} M^{0}((p, v)) \leq 1$ and

$-\forall t \in T \quad \bullet t \cap P \neq \emptyset$.

We define its symbolic unfolding

$$
U(N) \stackrel{\text { def }}{=}\left(B, P^{+}, P^{-}, E, \text { pre }_{U}, \text { post }_{U}, \Lambda, \lambda_{U}, \gamma_{U}, M_{U}^{0}\right)
$$

as follows: ( $B$ and $E$ are defined inductively)

1. $P^{+}$and $P^{-}$are the sets of interface places of $N$

2. initial conditions:

$\perp \bullet B$, with $\perp \stackrel{\text { def }}{=}\left\{(\perp, p) \mid p \in P, \exists v \in V, M^{0}(p, v)=1\right\}$

3. initial marking:

$$
\begin{aligned}
& \forall p \in P^{ \pm} \quad \forall v \in V \quad M_{U}^{0}(p, v) \stackrel{\text { def }}{=} M^{0}(p, v) \\
& \forall(\perp, p) \in \perp^{\bullet} \quad \forall v \in V \quad M_{U}^{0}((\perp, p), v) \stackrel{\text { def }}{=} M^{0}(p, v)
\end{aligned}
$$$$
\forall b \in B \backslash \perp^{\bullet} \quad \forall v \in V \quad M_{U}^{0}(b, v) \stackrel{\text { def }}{=} 0
$$

4. input and output of an event:

$$
\forall e=(C, t) \in E \quad\left\{\begin{array}{l}
\bullet \stackrel{\bullet \text { def }}{=} C \cup\left(\bullet t \cap P^{ \pm}\right) \quad \text { and } \\
e \stackrel{\bullet \text { def }}{=}\left\{(e, p) \mid p \in t^{\bullet} \cap P\right\} \cup\left(t^{\bullet} \cap P^{ \pm}\right)
\end{array}\right.
$$

5. firing modes (only those that are compatible with a valid coloring of $\lceil e\rceil$ ):

$$
\forall e=(C, t) \in E
$$

$$
\begin{aligned}
\gamma(e) \stackrel{\text { def }}{=}\left\{\left(\alpha \circ \phi_{N \mid \bullet e}, \beta \circ \phi_{N \mid e \bullet}\right) \mid(\alpha, \beta) \in \gamma(t) \wedge\right. \\
\left.\exists \text { Col } \in \text { valid_colorings }(\lceil e\rceil) \quad \operatorname{Col}_{\mid C}=\alpha \circ \phi_{N \mid C}\right\}
\end{aligned}
$$



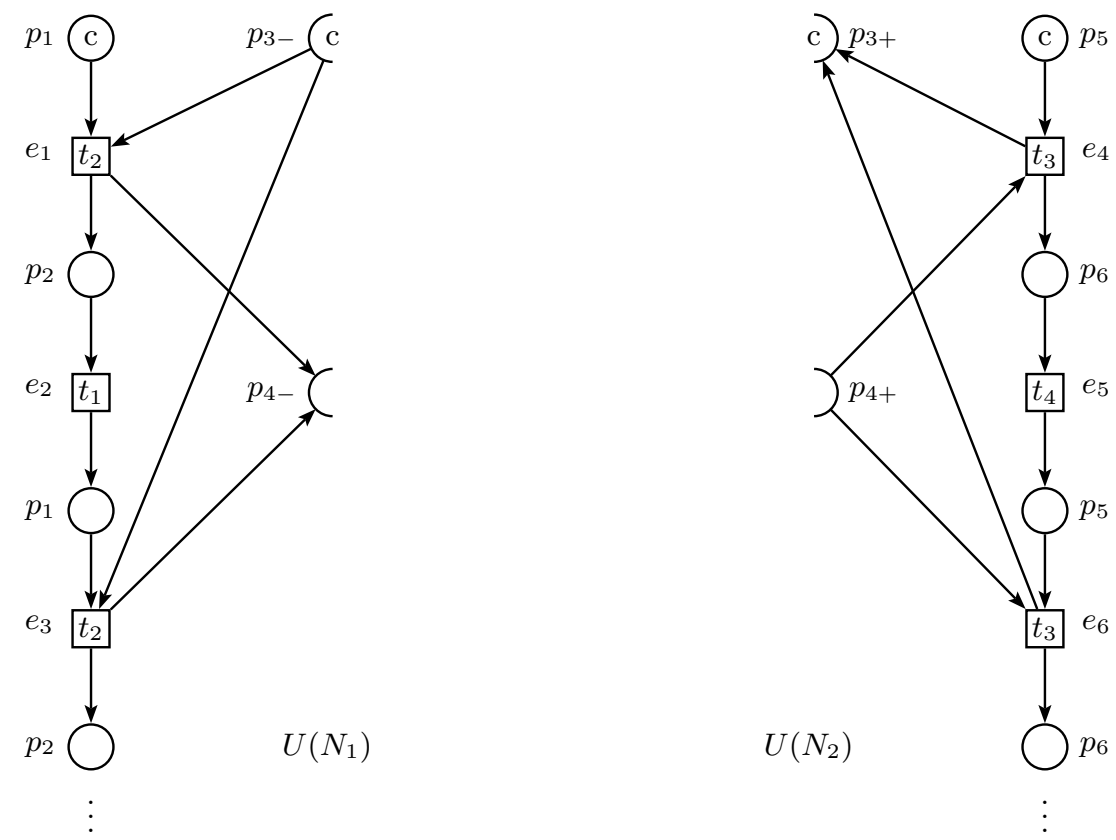

Fig. 4. The symbolic unfolding of each component of the colored puzzle net of Figure 1.

6. insertion of new events:

$$
\begin{aligned}
\forall e=(C, t) \in 2^{B} \times T, \quad e \in E \quad \text { iff } \\
\qquad \begin{array}{l}
\phi_{N \mid C} \text { is a bijection from } C \text { to } \bullet t \cap P \\
\nexists e_{1}, e_{2} \in\lceil e\rceil \quad e_{1} \neq e_{2} \wedge \bullet e_{1} \cap \bullet e_{2} \cap B=\emptyset \\
\left.\exists(\alpha, \beta) \in \gamma(e) \quad \exists C o l \in \text { valid_colorings }(\lceil e\rceil) \quad C_{0}\right) l_{\mid C}=\alpha_{\mid C}
\end{array}
\end{aligned}
$$

7. insertion of new conditions created by an event:

$\forall e \in E \quad e^{\bullet} \backslash P^{ \pm} \subseteq B$

8. labels:

$\Lambda$ is the set of labels of $N$;

$\forall x \in B \cup P^{ \pm} \quad \lambda_{U}(x)=\lambda\left(\phi_{N}(x)\right)$

Proposition 2. $U(N)$ is a symbolic occurrence net, and the folding $\phi_{N}$ : $U(N) \rightarrow N$ is a morphism.

See the proof in [8].

Theorem 4 (symbolic unfolding). The $U$ functor establishes a coreflection between the category of (unfoldable) colored puzzle nets and the full subcategory of symbolic occurrence nets.

Proof sketch. We have to prove the universal property of symbolic unfoldings: Let $\phi$ be a morphism from a symbolic occurrence net $O$ to an unfoldable net $N$. There exists a unique morphism $\psi: O \rightarrow U(N)$ such that $\phi=\phi_{N} \circ \psi$. We prove easily that if $\psi$ exists, it is unique and defined as: 


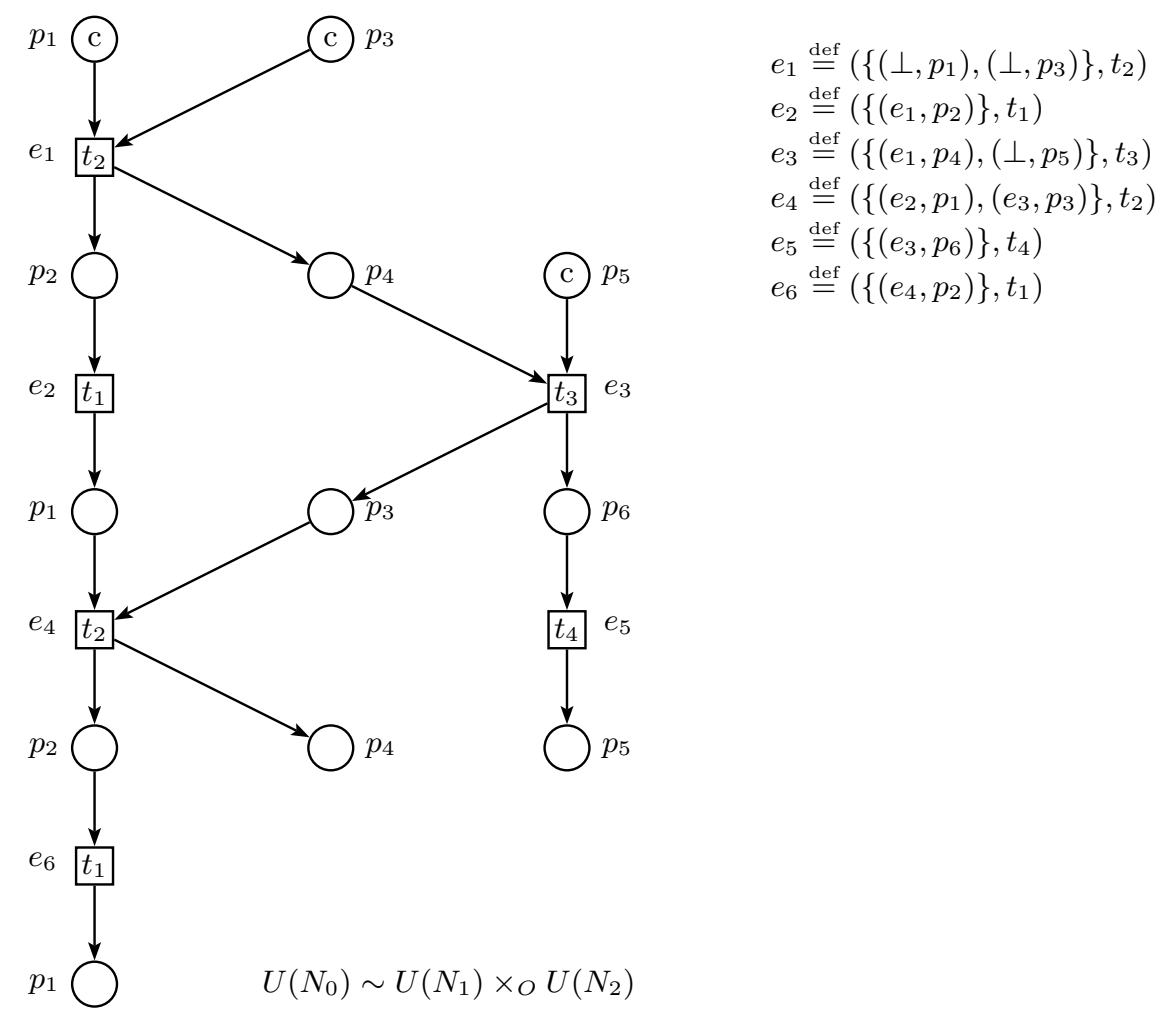

Fig. 5. The symbolic unfolding of the colored puzzle net $N_{0}=N_{1} \times N_{2}$ of Figure 1 .

$-\phi(x) \in P^{ \pm} \Longrightarrow \psi(x) \stackrel{\text { def }}{=} \phi(x)$

$-\psi(x)=*$ iff $\phi(x)=*$

$-\psi(e) \stackrel{\text { def }}{=}\left(\psi(\bullet e) \backslash P^{ \pm}, \phi(e)\right)$ if $\phi(e) \neq *$

$-\phi(b) \in P \Longrightarrow \begin{cases}\psi(b) \stackrel{\text { def }}{=}(\perp, \phi(b)) & \text { if } \bullet b=\perp \\ \psi(b) \stackrel{\text { def }}{=}(\psi(\bullet b), \phi(b)) & \text { otherwise }\end{cases}$

It remains to show that $\psi$ is a morphism from $O$ to $U_{N}$. The difficult part is to show that $\psi$ maps the events of $O$ to valid events of $U(N)$.

By the same arguments as for the expansion, one derives immediately the existence of a product in the subcategory of symbolic occurrence nets, given by $O_{1} \times_{O} O_{2} \stackrel{\text { def }}{=} U\left(O_{1} \times O_{2}\right)$. And, again, product is preserved by the symbolic unfolding functor:

$$
U\left(N_{1} \times N_{2}\right) \sim U\left(N_{1}\right) \times_{O} U\left(N_{2}\right)
$$

which was the announced result. This is illustrated in Figure 5. Notice that the factored form on the right hand side is by nature more compact than the symbolic unfolding of the product, since interface places between $N_{1}$ and $N_{2}$ are not expanded. 


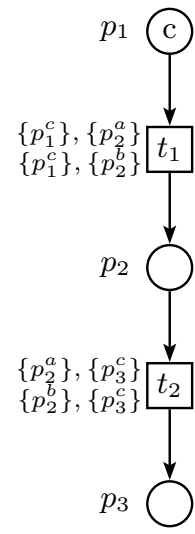

$N \sim U(N)$

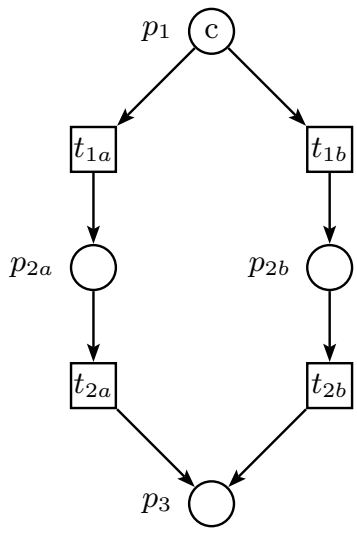

$\operatorname{Exp}(N)$

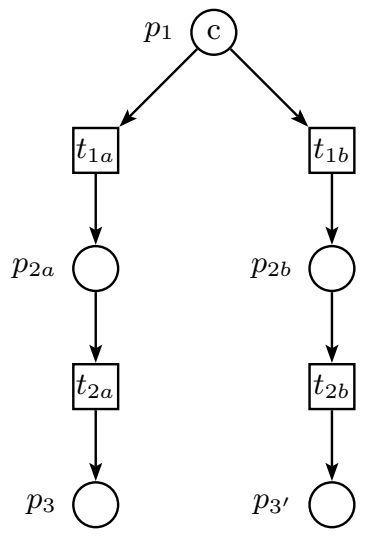

$\operatorname{Exp}_{O}(U(N)) \sim U(\operatorname{Exp}(N))$

Fig. 6. A colored Petri net $N$, which is isomorphic to its unfolding $U(N)$, the expansion $\operatorname{Exp}(N)$ of $N$ and the expansion $\operatorname{Exp}_{O}(U(N))$ of the unfolding.

\subsection{Expanded Unfoldings and Expansion of Colored Occurrence Nets}

Remark that the symbolic unfolding of an expanded puzzle net is an expanded occurrence net. Actually, when applied to expanded puzzle nets, our definition of symbolic unfolding matches the usual definition of unfoldings for low-level Petri nets [24] (up to interface places). This object forms a coreflection from expanded nets to expanded occurrence nets, and we call it the expanded unfolding.

We are looking for a relation between the symbolic unfolding and the expanded unfolding of a colored puzzle net. The idea is that expanding the symbolic unfolding should yield the expanded unfolding. But actually the expansion of an occurrence net is not an occurrence net in general. This fact is illustrated in Figure 6, where transition $t_{1}$ of $N$ produces either a token of color $a$ or $b$ in place $p_{2}$, and transition $t_{2}$ consumes it anyway and produces a token of color $c$ in place $p_{3}$. If $U(N)$ is expanded as a net, then the two versions of transition $t_{3}$ converge to the same place $p_{3}$, which is not suitable for an occurrence net.

The correct expansion functor $\operatorname{Exp}_{O}$ for occurrence nets is defined naturally as $\operatorname{Exp}_{O}(O)=U(\operatorname{Exp}(O))$. Composing the two coreflections built in the previous sections allows one to establish one more between the category of symbolic occurrence nets and the category of expanded occurrence nets, the former being viewed as a subcategory of colored puzzle nets (see Fig. 7). In addition, one has that the expanded unfolding $U(\operatorname{Exp}(N))$ is isomorphic to the expansion by $\operatorname{Exp}_{O}$ of its symbolic unfolding $U(N)$, that is $U(\operatorname{Exp}(N)) \sim \operatorname{Exp}_{O}(U(N))$.

All this results in the commutative diagram in Fig. 7, that displays the four categories derived from colored puzzle nets by expansion and by symbolic 


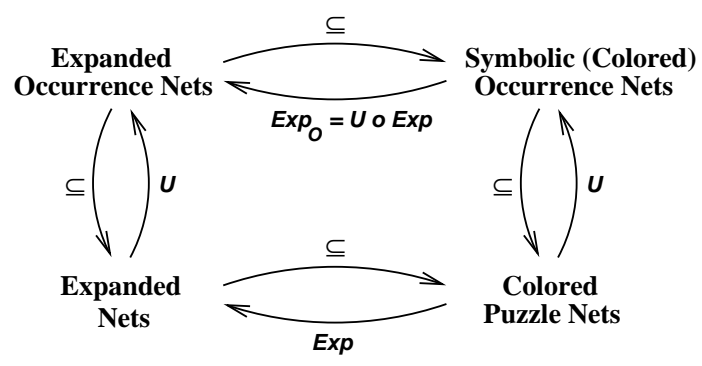

Fig. 7. Coreflections between categories derived from colored puzzle nets by symbolic unfolding and by expansion.

unfolding. The coreflections illustrated in this figure naturally transport categorical limits. For example, for two symbolic occurrence nets $O_{1}, O_{2}$, one has $\operatorname{Exp}_{O}\left(O_{1} \times_{O} O_{2}\right) \sim \operatorname{Exp}_{O}\left(O_{1}\right) \times_{E O} \operatorname{Exp}_{O}\left(O_{2}\right)$ where the product $\times_{E O}$ in the category of expanded occurrence nets is obtained by applying $\operatorname{Exp}_{O}$ to the product $x_{O}$ in the category of symbolic occurrence nets.

\section{Conclusion}

We have studied the unfoldings of colored puzzle nets, a formalism of high-level Petri nets using the popular composition mechanism based on shared places. An adequate categorical framework has been proposed for this family of nets, based on run-preserving morphisms. Symbolic unfoldings have been also adapted to colored puzzle nets, and related to previous notions of unfoldings for low-level nets, through the notion of expansion. In this adequate categorical framework, we have also illustrated an important property of the symbolic unfolding operation, namely that it commutes with product. The factorization property of unfoldings forms the basis of distributed processing methods for distributed systems (for example distributed diagnosis). We will now explore the interest of symbolic unfoldings for this purpose.

Let us mention that all derivations are presented for the family of colored puzzle nets, because we are convinced of the practical interest of composing nets via shared places. However, the same results remain valid with more ordinary categories of colored nets, where composition is performed by synchronizing transitions carrying identical labels.

\section{References}

1. P. Baldan, T. Chatain, S. Haar, and B. König. Unfolding-based diagnosis of systems with an evolving topology. In CONCUR, volume 5201 of $L N C S$, pages 203-217. Springer, 2008.

2. P. Baldan, A. Corradini, H. Ehrig, R. Heckel, and B. König. Compositional semantics for open Petri nets based on deterministic processes. Technical report, University of Pisa, Tech. Rep. TR-01-21, 2001. 
3. P. Baldan, A. Corradini, H. Ehrig, and B. König. Open Petri nets: Nondeterministic processes and compositionality. In ICGT, volume 5214 of $L N C S$, pages 257-273. Springer, 2008.

4. P. Baldan, A. Corradini, and U. Montanari. Contextual Petri nets, asymmetric event structures, and processes. Information and Computation, 171(1):1-49, 2001.

5. E. Best, R. Devillers, and M. Koutny. The box algebra $=$ Petri nets + process expressions. Inf. Comput., 178(1):44-100, 2002.

6. E. Best, H. Fleischhack, W. Fraczak, R. P. Hopkins, H. Klaudel, and E. Pelz. A class of composable high level Petri nets with an application to the semantics of $B(P N)^{2}$. In Application and Theory of Petri Nets, volume 935 of $L N C S$, pages 103-120. Springer, 1995.

7. E. Best, W. Fraczak, R. P. Hopkins, H. Klaudel, and E. Pelz. M-nets: An algebra of high-level Petri nets, with an application to the semantics of concurrent programming languages. Acta Inf., 35(10):813-857, 1998.

8. T. Chatain and É. Fabre. Factorization properties of symbolic unfoldings of colored Petri nets. Research Report LSV-10-07, Laboratoire Spécification et Vérification, ENS Cachan, France, April 2010.

9. T. Chatain and C. Jard. Symbolic diagnosis of partially observable concurrent systems. In FORTE, volume 3235 of LNCS, pages 326-342, 2004.

10. H. Ehrig, K. Hoffmann, K. Gabriel, and J. Padberg. Composition and independence of high-level net processes. Electr. Notes Theor. Comput. Sci., 242(2):59-71, 2009.

11. H. Ehrig, K. Hoffmann, J. Padberg, P. Baldan, and R. Heckel. High-level net processes. In Formal and Natural Computing, volume 2300 of LNCS, pages 191219. Springer, 2002.

12. J. Engelfriet. Branching processes of Petri nets. Acta Inf., 28(6):575-591, 1991.

13. E. Fabre. On the construction of pullbacks for safe Petri nets. In ICATPN, volume 4024 of $L N C S$, pages 166-180. Springer, 2006.

14. J. F. Groote and M. Voorhoeve. Operational semantics for Petri net components. Theor. Comput. Sci., 379(1-2):1-19, 2007.

15. K. Jensen. Coloured Petri nets: basic concepts, analysis methods and practical use. Springer-Verlag, 1995.

16. V. Khomenko. Model Checking Based on Prefixes of Petri Net Unfoldings. PhD thesis, School of Computing Science, University of Newcastle upon Tyne, 2003.

17. V. Khomenko and M. Koutny. Branching processes of high-level Petri nets. In TACAS, volume 2619 of $L N C S$, pages 458-472. Springer, 2003.

18. E. Kindler. A compositional partial order semantics for Petri net components. In ICATPN, volume 1248 of LNCS, pages 235-252. Springer, 1997.

19. M. Koutny and E. Best. Operational and denotational semantics for the box algebra. Theor. Comput. Sci., 211(1-2):1-83, 1999.

20. J. Meseguer, U. Montanari, and V. Sassone. On the semantics of place/transition Petri nets. Mathematical Structures in Computer Science, 7(4):359-397, 1997.

21. M. Nielsen, L. Priese, and V. Sassone. Characterizing behavioural congruences for Petri nets. In CONCUR, volume 962 of $L N C S$, pages 175-189. Springer, 1995.

22. L. Priese and H. Wimmel. A uniform approach to true-concurrency and interleaving semantics for Petri nets. Theor. Comput. Sci., 206(1-2):219-256, 1998.

23. W. Reisig. Simple composition of nets. In Petri Nets, volume 5606 of LNCS, pages 23-42. Springer, 2009.

24. G. Winskel. Categories of models for concurrency. In Seminar on Concurrency, Carnegie-Mellon Univ., volume 197 of LNCS, pages 246-267, 1985. 